\title{
Application and Practice of Large Data in Plant Protection Unmanned Aerial Vehicle
}

\author{
Liang Xue
}

Suzhou Polytechnic Institute of Agriculture, Suzhou, Jiangsu, 215008

Keywords: Big Data: Plant Protection UAV: Application: Practice

\begin{abstract}
With the advent of large data age, large data is to quickly and effectively deal with massive data technology affects all walks of life, including large data in the application of plant protection UAVs and practice, can effectively improve the plant protection work in the massive data Index and processing speed. Therefore, this paper mainly aims at the application and practice of large data in the application and practice of large-scale data protection in unmanned aerial vehicles (UAVs), and the application and practice of large data in the application of plant protection and unmanned aerial vehicles. It is hoped that this paper can provide some reference and reference for the application and practice of large data on plant protection UAV.
\end{abstract}

\section{Introduction}

With the rapid development and progress of information science and technology in China today, large data era has come, big data (big data) refers to a certain period of time cannot be used with conventional software to capture and process data collection, the need for new processing mode to have more Strong decision-making, insight into the discovery of power and process optimization capabilities of the massive, high growth rate and diversified information assets. Large data with 5V features, Volume, Velocity, Variety, Value, and Veracity. Modern large data is to quickly and effectively deal with massive data technology affects all walks of life, including large data in the application of plant protection UAV on the application and practice, can effectively improve the plant protection work in the large amount of data index and processing speed, for real-time collecting, automatically transmitting the relevant data in plant protection, for example, the type of crop, the type of drug spraying pesticide, the type of pest and disease that exists in the crop, the concentration of pesticide spraying, the area of the crop to be sprayed, etc., thus establishing a huge plant protection database the deployment of data collection, transmission, processing, service, applied to one, to build intelligent, automated plant protection, the deployment of information, Work has a very important role.

\section{The Necessity of Application and Practice of Large Data in the Plant Protection UAV}

In the past, Chinese traditional plant protection work, generally mainly through the use of telescopes, or by plane to take the plane to take pictures, and then the image of the image upload and analysis process. However, this kind of artificial field monitoring and the way of plant protection is not only difficult to move, but also the cost is too high, often the actual detection of images is not accurate enough. And nowadays, especially in many developed countries abroad, through the large data in the application of plant protection UAV can be achieved through the wireless network to the plant protection detection platform, and then through the corresponding large data software system for rapid upload, Analysis and other processes, which can be largely to achieve the purpose of saving manpower and material resources. And through large data in the plant protection UAV on the application and practice can make plant protection better. For example, through the application and practice of large data on plant protection unmanned aerial vehicles and it is possible to predict the possibility of flooding and so on the ground and the river in advance, so as to realize the method of preparing the corresponding measures in the plant protection work. The large data system in the plant protection unmanned aerial vehicle can also help the ground to do a 
comprehensive and effective preparedness and response through the collection of the water content and the electricity consumption of the atmospheric clouds, so that the plant protection work can be more refined, quantified and rationalized as well as scientific.

\section{The Main Technology Applications in the Major Data Used in Plant Protection UAV}

With the current large-scale data in the application of plant protection UAV on the application and practice of more and more in-depth, a variety of large data theory and algorithm after another, how to use the optimal algorithm and theory to achieve plant protection UAV in the mass Data collection, processing, transmission, storage and other work, has become a modern large data in the plant protection UAV on the application and practice of the enormous challenges facing.

The Commonly Used Technology Theory of Large Data in the Application of Plant Protection UAV. Nowadays, foreign countries in Europe and the United States large data in the application of plant protection UAVs and practice is the most commonly used data activation theory. Data activation theory is mainly through the UAV in the computer environment for a spontaneous evolution, so as to improve the UAV large data storage organization, and the purpose of computing efficiency. Although the current technology in the context of the rapid development of science and technology in the world, a variety of large data environment based on the application and practice in the plant protection UAV in the technical theory after another, such as: Rhododendron-Download Runer storage system theory, blue gene Blue Gene / L storage system, metadata management theory, distributed multi-level cache management theory, distributed data layout theory, but the status of data activation theory is still unable to shake [1]. This is mainly due to the fact that data activation theory can maintain relative adaptability in the application and practice of plant protection UAVs in large data environment, and has some cognitive ability, for example, by integrating data activation theory The application of the plant protection unmanned aerial vehicle enables intelligent automatic identification of the data parameters such as the type of crop, the type of drug spraying the pesticide, the type of pest and disease in which the pesticide is present, the concentration of the pesticide sprayed, the area of the crop to be sprayed, and so on can be based on set a good pesticide formula, generate extremely accurate pesticide spraying system to ensure that plant protection work more smoothly and efficiently.

The Commonly Used Equipment Agreement of Large Data in the Plant Protection UAV Applications. Large data in the plant protection UAV applications commonly used types of device protocols are more, for now, commonly used device protocols include Modbus, TCP / IP protocol and TCP / UDP protocol.

Modbus is one of the earliest equipment protocols commonly used by Schneider, which is widely used in the application and practice of plant protection UAV. Modbus is a more commonly used and popularized protocol, mainly by intelligent terminal equipment Programmable logic controller and computer line connection made for large data in the application of plant protection UAV and practice play a more important role.

TCP / IP protocol (Transmission Control Protocol / Internet Protocol), is the largest data in the application of UAV applications in the most common, but also the most frequently used a device agreement, mainly due to most of the plant protection UAV Of the large data platform, which the network layer and the transport layer is mainly by the IP protocol and TCP protocol together. TCP / IP protocol in the application of large data in the plant protection UAV and the process can be found by a large number of information processing and transmission of possible problems in order to achieve once the problem will automatically send a signal to re-transmission purposes, making The data collected by all unmanned aerial vehicles, such as planting information, geographical location information, pest and disease information, field real-time climate information, field real-time temperature information, field real-time humidity information, pesticide library information, pesticide spraying information and other data information, UAV large data system which is safe and complete storage, and accurate transmission to the database [2].

TCP / UDP protocol (Transmission Control / User Datagram Protocol), as plant protection UAV large data platform in the transport layer is a more commonly used in a protocol, in the plant 
protection UAV large data system information processing process Reliable data transmission, TCP / UDP protocol through the data flow transmission reliability, effective flow control and other advantages, in the plant protection UAV large data system information processing process to establish a good channel, in order to achieve large amounts of data Transmission of high economic efficiency and high reliability.

\section{The Application and Practice of Plant Protection UAV Large Data Platform}

Although in recent years, Chinese plant protection UAV large platform for the construction and implementation of a breakthrough in the work, but compared to foreign plant protection UAV large platform for a wide range of applications and developed technology, China should have a certain Backward, therefore, in the plant protection UAV large data platform design and implementation work, our country can fully reference the foreign mature experience and methods, through the IEC61850 standard for plant protection UAV large data platform construction, and through the data more The organization of index and optimization of data processing technology, to a large extent, can be extremely effective in promoting the establishment of Chinese plant protection UAV large data platform to achieve the protection of agriculture and forestry plant unmanned aircraft, spraying pesticides, seeds, the work has a higher efficiency and better environmental protection, plant protection effect.

The Basic Hardware Structure of Plant Protection UAV Large Data Platform. With the rapid development of social economy and science and technology, people are now on the large data in the application of plant protection UAV technology and have been more and more demanding, through the large data in the application of plant protection UAV applications and practice of the research, to help Chinese plant protection work better to achieve intelligent. At present, the global technology and basic structure of the large data platform established in the application and practice of plant protection UAV can be described as diversified. However, IEC61850 has become a modern plant protection principle in the construction of the core basic structure of large data platform. UAV applications and practice in the common standards. Through the implementation of the IEC61850 standard in the large data platform for plant protection UAV, it can make the large data system of plant protection UAV become standardized, unified and transparent. For example, the Lockheed (Wuhan) UAV Scientific Research Institute Co., Ltd., through the use of IEC61850 standard in the plant protection UAV large data platform applications, making plant protection UAV large platform in the station control layer through intelligent Module interaction, network communication in the station control layer SNTP or MMS protocol and the interval when the information exchange configuration, once found any problems, it will automatically achieve the alarm signal upload, as well as analog, real-time status control information interaction And real-time data upload, etc., to facilitate the supervision of the plant protection UAV to achieve a comprehensive safety supervision and job scheduling and other aspects of the work. At the same time, the space in the large data platform of the plant protection UAV can play a role in protection and control. All data signal information, status information and action information, such as the speed of UAV operations, UAV Flight attitude, plant temperature and humidity parameters of the environment, etc., can be plant protection UAV large platform in the SV / GOOSE / 1588 and other fast message transmission mechanism in the process layer of the process interface transmission, and by the electronic Transformers and intelligent switches for automatic control. The basic module of IEC61850 standard, station control layer and interval layer in the large data platform of the plant protection unmanned aerial vehicle makes the basic data of agricultural data such as meteorological condition, pest and disease information, and can be collected and transferred by more cost-effective and more accurate way for plant protection UAV large data platform provides a more efficient and convenient hardware support [3].

Data Protection Technology for Plant Protection UAV Large Data Platform. In the data indexing technology used in the large data platform of the plant protection UAV, the data polymorphism organization indexing technology is one of the most commonly used indexing techniques. Data polymorphism organization indexing technology, in fact, is based on a discrete 
target indexing method, mainly for the target area within a specific set of points to search. Especially for the plant protection work often has coverage and search area and a wide range of characteristics, through the data polymorphism organization indexing technology in the plant protection UAV large data platform applications, can be achieved within a certain range of targets to be searched Probability has a relatively high effect. At the same time, due to data polymorphism organization indexing technology, especially for large data environment in the data quality, network connectivity, energy efficiency, network fault tolerance, algorithm complexity, algorithm accuracy, dynamics and compatibility The scalability of the network, the complexity of the implementation and other aspects of the requirements are low, and thus, it has become a large plant data platform for data protection technology used in the most practical and convenient data indexing technology

Data Processing Technology Used in Large Data Platform for Plant Protection UAV. Large data in the application and practice of plant protection UAV, the most important thing is how to deal with massive data information, how can we use the UAV sensor collected by the huge data flow, to ensure data integrity and reliability under the premise Effective processing, it is necessary to strengthen the large data in the application of plant protection UAV in the data processing technology. Through the comprehensive monitoring of the status information of all the data collected by the plant protection UAV in the large data environment, according to the different data sources, according to the different actual situation, the data acquisition module will provide the original raw data, the original data source includes fixed information such as the quantity and area of the crop, and a series of changes such as the number and type of pests and diseases. When the data extraction module obtains the data source, through the data source for simple filtering, data format standardization and other processing, and the processed data into the database, this time plant protection UAV data processing technology will database data in-depth analysis and classification, which according to different protocols to establish the corresponding data warehouse, through the data mining, can find the basic time to change the rules, and ultimately submitted to the UAV large data platform in the data processing system generation module. The resulting modules will be fully monitored for all data that may appear in the UAV large data platform. Once the indexed data is being monitored, the above steps are repeated and the above steps are repeated. This kind of data processing technology in the large data platform of plant protection unmanned aerial vehicles can realize the analysis, induction and excavation of data to a large extent, and provide powerful methods and means for the benign operation of plant protection work [4].

\section{Conclusion}

Large data in the application of plant protection UAV on the application and practice, can continuously improve the plant protection work in the massive data collection, processing, transmission, storage methods and methods to effectively ensure the plant protection data integrity and timeliness, to avoid large data in the application of plant protection UAV and the process of data indexing, collection, processing and other possible data loss or data overflow occurred in the situation. Therefore, the study of the application and practice of large data on plant protection unmanned aerial vehicles has very important positive significance.

\section{References}

[1] Cai Hongzhao, Mou Tao, Liu Jingchao, etc. A design of a flight control system for agricultural sprayed unmanned aerial vehicles[J]. Changchun: Agriculture and Technology, 2015: 41-42.

[2] Zhou Zhiyan, Zang Ying, Luo Xiwen, etc. Chinese agricultural aviation plant protection industry technology innovation and development strategy[J]. Beijing: Journal of Agricultural Engineering, 2014: 123-142.

[3] Song Yu. Unmanned helicopter plant protection technology research progress[J]. Hefei: Modern Agricultural Science and Technology, 2016: 136-138. 
[4] Zhu Yanzhi. Small agricultural unmanned helicopter flight control technology research [D]. Nanjing: Nanjing University of Aeronautics and Astronautics, 2015. 